\title{
MATHEMATICAL NOTE
}

\section{A NOTE ON SOME THEOREMS FOR ORDINARY DIFFERENTIAL EQUATIONS}

\author{
by YIK-HOI AU-YEUNG \\ (Received 22nd April 1965)
}

1. In his first stability theorem [(1), p. 259], Liapounoff has proved the following fact: Let

$$
\frac{d y}{d t}=Y(t, y)
$$

where $Y$ is continuous on the region

$$
R^{*}: \quad t \geqq T,|y| \leqq H,
$$

where $T$ and $H(>0)$ are constants, and $Y(t, 0)=0$ for $t \geqq T$. If for $\left({ }^{*}\right)$ there exists a continuously differentiable positive definite function $V(t, y)$ such that $V(t, 0)=0$ for $t \geqq T$ and

$$
\frac{\partial V}{\partial t}+\frac{\partial V}{\partial y} Y \leqq 0
$$

then the trivial solution of $\left(^{*}\right)$ is stable. Now if we make a transformation $x=1 / t$, then Liapounoff's second method can be used to study the behaviour of the solutions of the equation

$$
\frac{d y}{d x}=f(x, y)
$$

where $f$ is defined and continuous on the region

$$
R: 0<x \leqq a, 0 \leqq y \leqq b,
$$

and $f(x, 0)=0$ for $0<x \leqq a$. In particular, Theorem 1 below can be obtained in this way. However, since the direct proof is quite short, we give this as well.

\section{Some theorems for ordinary differential equations}

Theorem 1. If for (1) there exists a function $g(x) \in C((0, a])$ satisfying $0 \leqq g(x) \leqq b, g(a)>0$, and a function $V(x, y)$, defined and continuously differentiable on the region

$$
R_{1}: 0<x \leqq \underset{165}{a, 0 \leqq y \leqq g(x)},
$$


such that

$$
\begin{gathered}
\inf _{\{0<x \leqq a, g(x) \neq 0\}} V(x, g(x))>0, \\
V(a, 0)=0, \\
\frac{\partial V}{\partial x}+\frac{\partial V}{\partial y} f(x, y) \geqq 0,
\end{gathered}
$$

then there is a $\delta>0$ such that any solution $y=y(x)$ of $(1)$ in region $R_{1}$ satisfying $y(a)=k$ with $0 \leqq k \leqq \delta$ can be continued to the left as a solution of (1) in $R_{1}$ defined on $(0, a]$.

Proof. Let $\gamma=\inf _{\{0<x \leqq a, g(x) \neq 0\}} V(x, g(x))$, then by (A) we see that $\gamma>0$. By (B) there is $\delta>0(\delta \leqq g(a))$ such that

$$
V(a, k)<\gamma,
$$

for all $k$ satisfying $0 \leqq k \leqq \delta$. Take a solution $y=y(x)$ of (1) in region $R_{1}$ satisfying $y(a)=k$ with $0 \leqq k \leqq \delta$. Suppose this solution cannot be continued to the left in $R_{1}$ at a point $x_{0}$ in $(0, a)$; then, by the continuation theorem (for example, see (2), p. 15) and the fact that $y=0$ is a solution of (1) in $R_{1}$ defined on $(0, a]$, we have $y\left(x_{0}\right)=g\left(x_{0}\right) \neq 0$. By (C) we have

$$
\frac{d V(x, y(x))}{d x} \geqq 0
$$

for all $x \in\left(x_{0}, a\right)$. Therefore,

$$
V(a, y(a)) \geqq V\left(x_{0}, y\left(x_{0}\right)\right) .
$$

But on the other hand by (2) and (A), we have

and

$$
V(a, y(a))=V(a, k)<\gamma
$$

$$
V\left(x_{0}, y\left(x_{0}\right)\right)=V\left(x_{0}, g\left(x_{0}\right)\right) \geqq \gamma .
$$

This is a contradiction. Thus Theorem 1 is proved.

As an immediate consequence of Theorem 1, we have the following theorem for ordinary differential equations (this can also be obtained directly from the result quoted in $\$ \mathbf{1}$ ).

Theorem 2. If for (1) there exists a function $g(x) \in C([0, a])$, satisfying $g(0)=0$ and $0<g(x) \leqq b$ for all $x \in(0, a]$, and a function $V(x, y)$, defined and continuously differentiable on $R_{1}$, such that

$$
\begin{gathered}
\inf _{0<x \leqq a} V(x, g(x))>0, \\
V(x, 0)=0 \\
\frac{\partial V}{\partial x}+\frac{\partial V}{\partial y} f(x, y) \geqq 0,
\end{gathered}
$$


then for any $\alpha>0(\alpha \leqq a)$ there are infinitely many solutions of (1) in $R_{1}$ defined on $[0, \alpha]$ passing through $(0,0)$.

In particular, if we take $g(x)=x^{\beta}$, where $\beta$ is a positive real number, and take $V(x, y)=y / x^{\beta}$, then it is obvious that conditions $\left(\mathrm{A}^{\prime}\right)$ and $\left(\mathrm{B}^{\prime}\right)$ are satisfied. In this case, condition ( $\left.C^{\prime}\right)$ is equivalent to the condition that $f(x, y) \geqq \beta y / x$. Hence we have the following corollary.

Corollary. If for (1), there exist $a \beta>0$ and an $a_{1}$ satisfying $0<a_{1} \leqq a$ and $a_{1}^{\beta} \leqq b$ such that in the region

$$
R_{2}: 0<x \leqq a_{1}, 0 \leqq y \leqq x^{\beta}
$$

we have $f(x, y) \geqq \beta y / x$ then for any $\alpha>0\left(\alpha \leqq a_{1}\right)$ there are infinitely manysolutions of (1) in $R_{2}$ defined on $[0, a]$ passing through $(0,0)$.

\section{An example}

Consider the following equation (3)

where

$$
\frac{d y}{d x}=f(x, y)
$$

$$
f(x, y)=\left\{\begin{array}{cl}
(1+\varepsilon) y / x & \text { for } 0<y<x^{1+\varepsilon} \\
(1+\varepsilon) x^{\varepsilon} & \text { for } y \geqq x^{1+\varepsilon} \\
0 & \text { for } y \leqq 0
\end{array}\right.
$$

where $x \geqq 0$ and $\varepsilon$ is a positive constant. In the region

$$
R_{3}: 0<x \leqq a, 0 \leqq y \leqq x^{1+\varepsilon},
$$

where $a$ is any positive real number, it is obvious that $f(x, y) \geqq(1+\varepsilon) y / x$. Hence by the Corollary we see for any $a>0$ there are infinitely many solutions. of (3) in $R_{3}$ defined on $[0, a]$ passing through $(0,0)$.

\section{REFERENCES}

(1) A. Liapounoff, Problème Général de la Stabilité du Mouvement (Princeton, 1947).

(2) E. A. Coddington and N. Levinson, Theory of Ordinary Differential Equations (McGraw-Hill, 1955).

(3) O. Perron, Eine Hinreichende Bedingung für die Unität der Lösung. von Differentialgleichungen erster Ordnung, Math. Z. 28 (1928), 216-219.

UNIVERSITY OF HONG KONG 